\title{
The Ecology and Evolutionary Dynamics of Meiotic Drive
}

ARTICLE in TRENDS IN ECOLOGY \& EVOLUTION · FEBRUARY 2016

Impact Factor: $16.2 \cdot$ DOI: 10.1016/j.tree.2016.02.001

READS

46

22 AUTHORS, INCLUDING:

Wolfgang Forstmeier

Max Planck Institute for Ornithology

83 PUBLICATIONS 2,196 CITATIONS

SEE PROFILE

Gerald S Wilkinson

University of Maryland, College Park

136 PUBLICATIONS 5,512 CITATIONS

SEE PROFILE
Nina Wedell

University of Exeter

139 PUBLICATIONS $\quad 6,404$ CITATIONS

SEE PROFILE

Tom A. R. Price

University of Liverpool

38 PUBLICATIONS 353 CITATIONS

SEE PROFILE 


\section{Review}

\section{The Ecology and Evolutionary Dynamics of Meiotic Drive}

Anna K. Lindholm, ${ }^{1, \star}$ Kelly A. Dyer, ${ }^{2}$ Renée C. Firman, ${ }^{3}$ Lila Fishman, ${ }^{4}$ Wolfgang Forstmeier, ${ }^{5}$ Luke Holman, ${ }^{6}$ Hanna Johannesson, ${ }^{7}$ Ulrich Knief, ${ }^{5}$ Hanna Kokko, ${ }^{1}$ Amanda M. Larracuente, ${ }^{8}$ Andri Manser, ${ }^{1}$ Catherine Montchamp-Moreau, ${ }^{9}$ Varos G. Petrosyan, ${ }^{10}$ Andrew Pomiankowski, ${ }^{11}$ Daven C. Presgraves, ${ }^{8}$ Larisa D. Safronova, ${ }^{10}$ Andreas Sutter, ${ }^{1}$ Robert L. Unckless, ${ }^{12}$ Rudi L. Verspoor, ${ }^{13}$ Nina Wedell, ${ }^{14}$ Gerald S. Wilkinson, ${ }^{15}$ and Tom A.R. Price ${ }^{13}$

Meiotic drivers are genetic variants that selfishly manipulate the production of gametes to increase their own rate of transmission, often to the detriment of the rest of the genome and the individual that carries them. This genomic conflict potentially occurs whenever a diploid organism produces a haploid stage, and can have profound evolutionary impacts on gametogenesis, fertility, individual behaviour, mating system, population survival, and reproductive isolation. Multiple research teams are developing artificial drive systems for pest control, utilising the transmission advantage of drive to alter or exterminate target species. Here, we review current knowledge of how natural drive systems function, how drivers spread through natural populations, and the factors that limit their invasion.

\section{The Battle for Transmission}

One of the few rules in biology is Mendel's law of equal segregation: the two copies of each gene and/or chromosome in a diploid organism are transmitted with equal probability to its offspring. Although often taken for granted, it is increasingly clear that equal segregation is a fragile détente in a world of constant intragenomic competition (see Glossary) for passage to the next generation. Such conflict plays out in the arenas of meiosis and gametogenesis, and results in meiotic drive [1], the biased transmission of a gene or chromosome against its alternative (Box 1). Because selection on meiotic drive elements operates at a level below that of the individual, drivers can spread through populations even if they reduce organism fitness [2]. By the same process, recently developed synthetic drive elements, which are currently still confined to laboratories, have the potential to rapidly modify genomes in wild populations [3]. Both natural and synthetic drive systems can have profound ecological, evolutionary, and genomic consequences.

\section{Meiotic Drive Systems in Nature}

In this review, we explore the ecological and evolutionary dynamics of natural meiotic drive systems. We focus on three types of drive: female meiotic drive, male meiotic drive (sperm killers), and drive in haploid spores (spore killers, Box 1). However, meiotic drive can

\section{Trends}

Both naturally occurring and synthetic 'meiotic drivers' violate Mendel's law of equal segregation and can rapidly spread through populations even when they reduce the fitness of individuals carrying them.

Synthetic drivers are being developed to spread desirable genes in natural populations of target species. How ecology influences the population dynamics of meiotic drivers is important for predicting the success of synthetic drive elements.

An enduring puzzle concerns why some meiotic drivers persist at stable, intermediate frequencies rather than sweeping to fixation.

Drivers can have a wide range of consequences from extinction to changes in mating system.

${ }^{1}$ Department of Evolutionary Biology and Environmental Studies, University of Zurich, Zurich, Switzerland ${ }^{2}$ Department of Genetics, University of Georgia, Athens, GA 30602, USA ${ }^{3}$ Centre for Evolutionary Biology, School of Animal Biology, University of Western Australia, Perth, Western Australia 6009, Australia ${ }^{4}$ Division of Biological Sciences, University of Montana, Missoula, MT, 59812, USA 


\section{Trends in Ecology \& Evolution}

encompass a broad range of systems we do not discuss, including supernumerary B chromosomes, zygote killers, and paternal genome eliminators.

Female meiotic drive occurs when homologous chromosomes are differentially transmitted to the egg during meiosis. In plants and animals, female meiosis is asymmetric, with only one of the four meiotic products becoming an egg or, in plants, a megagametophyte ([4], Box 1). Any chromosomal variant that biases its own segregation (e.g., by preferentially associating with and moving toward the egg pole at Meiosis I) will be transmitted to more than half of the maturing eggs. Although this bias does not necessarily reduce the production of eggs (as only one egg matures per meiosis), the fitness of other alleles at the same locus, that do not bias transmission, and alleles linked to them, is reduced. Such meiotic drivers could reduce the fitness of individuals that carry them, if the driving variant is genetically linked to deleterious mutations or has deleterious pleiotropic effects.

Male meiotic drive takes multiple forms - some at least partially meiotic, some entirely postmeiotic - but all involve a driving element that prevents maturation or function of sperm that do not contain it. Because haploid sperm within a single ejaculate compete to fertilise the same pool of eggs, disabling noncarrier sperm results in transmission of the driving element to more than half of the functional gametes and resulting offspring ([5], Box 1). However, disabling noncarrier sperm often reduces fertility [6].

Spore drive in fungi, in which the products of meiosis are packaged together in an ascus, operates via similar mechanisms. Spores with one haploid genotype will kill or disable spores of the alternative haplotype ([7], Box 1). If spores disperse long distances sibling spores are unlikely to compete and killing them will not increase the killer's fitness. However, spore killing can be beneficial if there is local resource competition.

Exciting progress has been made in dissecting the genetic and cellular mechanisms of multiple drive systems that span eukaryotic diversity (Box 1). However, we are still in the early stages of understanding how these genetic systems interact with ecology to shape the dynamics of drivers in natural populations. The fate of a meiotic driver depends on the costs of transmission bias, the mating system, environmental factors, and population and geographic structure that affect the fitness of its carriers. These interactions might then affect how drivers contribute to genetic and phenotypic variation within and among populations, potentially contributing to speciation [8]. On a larger timescale, coevolution between drive elements and suppressors
${ }^{5}$ Department of Behavioural Ecology and Evolutionary Genetics, Max Planck Institute for Ornithology, 82319 Seewiesen, Germany

${ }^{6}$ Division of Ecology, Evolution and Genetics, Research School of Biology, Australian National University,

Canberra, Australia

${ }^{7}$ Department of Organismal Biology, Uppsala University, Norbyvägen 18D, 75236 Uppsala, Sweden

${ }^{8}$ Department of Biology, University of Rochester, Rochester, NY, USA

${ }^{9}$ Évolution Génomes Comportement et Ecologie, CNRS, IRD, Univ. Paris-Sud, Université Paris-Saclay, Gif-sur-Yvette, France

${ }^{10}$ Severtsov Institute of Ecology and

Evolution, Russian Academy of

Sciences, Moscow 119071, Russia

${ }^{11}$ Department of Genetics, Evolution and Environment, University College London, Gower Street, London WC1E 6BT, UK

${ }^{12}$ Department of Molecular Biology and Genetics, Cornell University, Ithaca, NY, USA

${ }^{13}$ Institute of Integrative Biology,

University of Liverpool, Liverpool L69 7ZB, UK

${ }^{14}$ Biosciences, University of Exeter, Cornwall Campus, Penryn, Cornwall TR10 9FE, UK

${ }^{15}$ Department of Biology, University of Maryland, College Park, MD, USA

${ }^{*}$ Correspondence: anna.lindholm@ieu.uzh.ch (A.K. Lindholm).
Box 1. Definition, Mechanisms, and Species

Meiotic drive occurs when alleles, haplotypes, or chromosomes subvert mechanisms of fair segregation to obtain greater than Mendelian transmission at the expense of homologues. Sandler and Novitski [1] first used the term 'meiotic drive' to describe biased transmission that results as 'a consequence of the mechanics of the meiotic divisions'. For instance, in taxa with asymmetric female meiosis, structural elements of chromosomes - for example, centromeres, telomeres, and heterochromatic neocentromeres ('knobs') - can compete for inclusion in the gamete and hence transmission to subsequent generations, with failing chromosomes discarded into the polar bodies. Examples of drive through female meiosis have been observed in mice [22,36], maize [80], and monkeyflowers ([35], Figure IA). However, 'meiotic drive' is often used in a broader sense to include biased transmission resulting from a variety of premeiotic, meiotic, and postmeiotic events during gametogenesis [17]. In males, for instance, drive elements can achieve biased transmission by killing sperm that lack the element (Figure IB). These gametic drivers typically involve a drive locus and a target locus. They can occur on autosomes - as in the mouse $t$ haplotype [56] and the fruit fly Segregation Distorter [31] - or on sex chromosomes, causing distorted sex ratios among progeny - as in Silene flowering plants [81], stalk-eyed flies [82], mosquitoes [17], and many Drosophila species [17]. Finally, in fungi a heterozygous cross between strains carrying a spore killer allele and a sensitive allele results in elimination of haploid ascospores that lack the spore killer allele ([7], Figure IC). Spore killer genetics can involve a single locus [83], or be complex, involving multiple loci [33]. Even this brief summary highlights that selfish drive elements gain transmission advantages through diverse genetic mechanisms across the eukaryotes. 


\section{Trends in Ecology \& Evolution}

\section{CellPress}

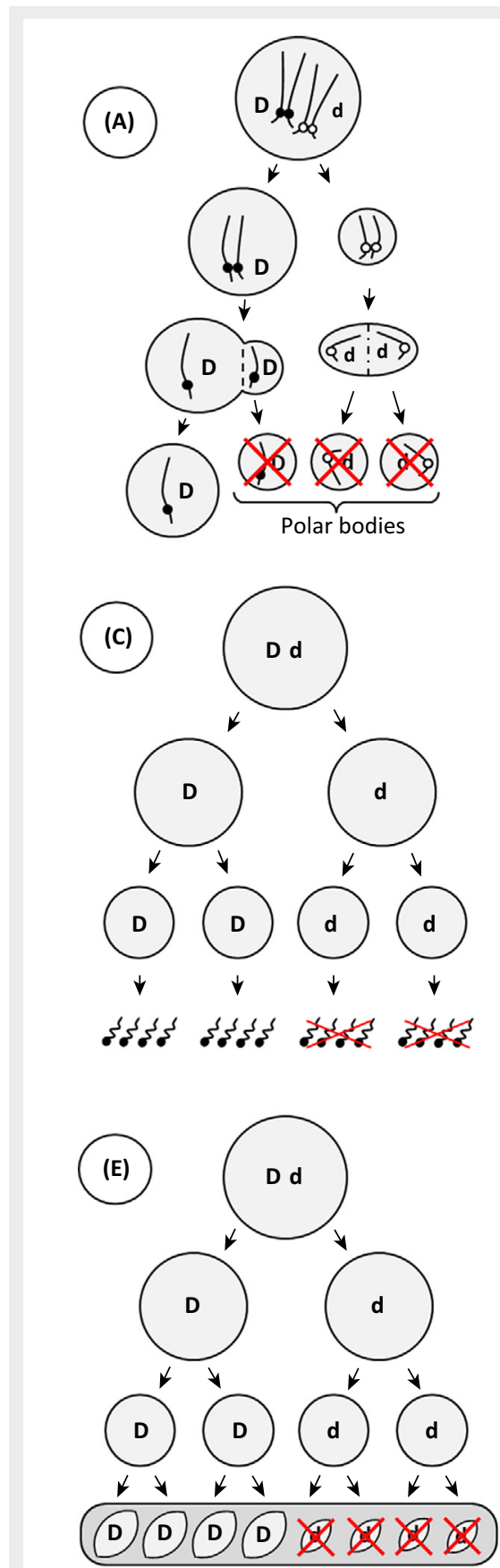

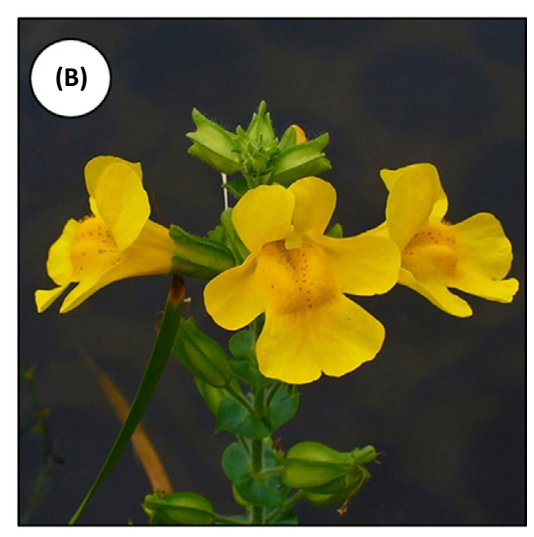
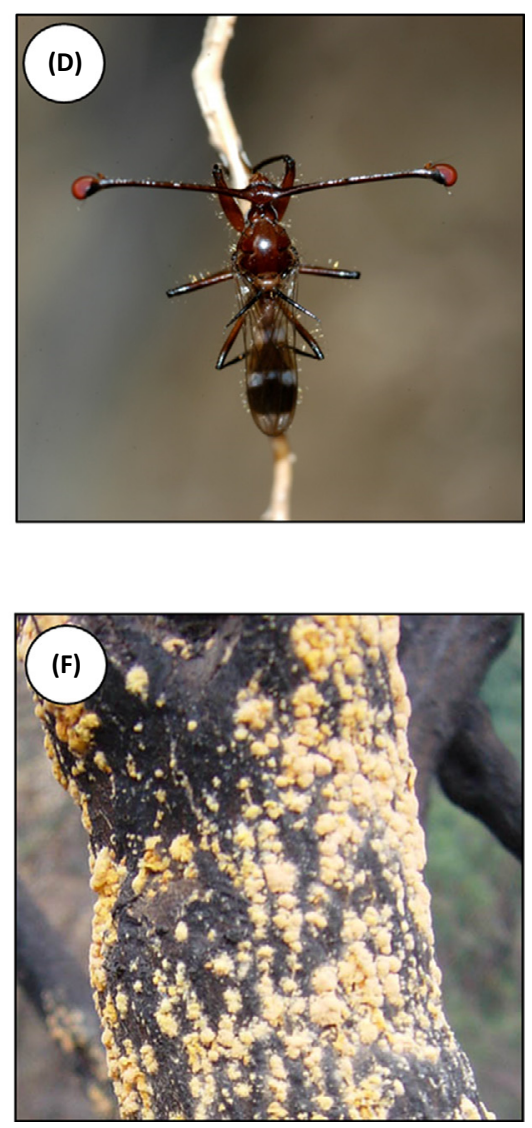

Trends in Ecology \& Evolution

Figure I. Meiotic Drive. The first column shows schematics of three types of meiotic drive, with the second column showing a species that carries that drive system. (A) Female gametogenesis: driving chromosomes relegate rival chromosomes to the polar bodies. The polar bodies are lost, while the drive chromosome enters the egg. (B) Female drive occurs in monkeyflowers. (C) Male gametogenesis: driving chromosomes (' $D$ ') cause sperm that carry the rival chromosome ('d') to die. (D) Sperm killing segregation distortion occurs in stalk-eyed flies. (E) Fungal spore production. Similar to male drivers, spore killers cause the death of spores that carry rival chromosomes. $(F)$ A spore killing system found in Neurospora fungi. Images: (B) Lila Fishman, (D) Gerald Wilkinson, and (F) Hanna Johannesson.

\section{Glossary}

Ascus: the sexual cell in fungi that undergoes meiosis to produce spores, typically eight.

Autosomal drivers: transmission distorters located on autosomal chromosomes.

Centromere: the part of the chromosome attached to the spindle during cell division that allows chromosomes to separate during meiosis.

CRISPR-Cas9: a genome editing technique involving a Cas9 nuclease, originally isolated from bacteria, that cuts target sites in the genome specified by complementary guide RNAs.

Drive suppressors: factors that reduce the transmission rate of a driver.

Enhancers: genes that increase the transmission rate of a driver. Female meiotic drive: biased transmission that arises during asymmetric female meiosis.

Fisherian sex ratio selection: theory predicting 1:1 male/female sex ratios because the fitness of the rarer sex is higher, all else being equal. Homing endonuclease genes: transmission distorters that insert themselves onto the homologous chromosome during DNA repair, converting a heterozygote into a homozygote for the element. Intragenomic competition and conflict: the conflict between elements of the genome when the action of one reduces the transmission of the other, encompassing meiotic drive, selfish endosymbionts, transposable elements, homing endonucleases, and many others.

Karyotype: the number and largescale structure of chromosomes of an individual.

Male meiotic drive: biased transmission that arises during male gamete production.

Meiotic drivers: allelic variants that manipulate gamete production to ensure they are transmitted to more than a fair Mendelian proportion of gametes.

Polyandry: female mating with multiple males.

Postzygotic (nondrive) distortion mechanisms: selection on zygotes, for example, the natural death of low fitness zygotes.

\section{Segregation Distorter: an}

autosomal male driver in Drosophila 


\section{Trends in Ecology \& Evolution}

\section{Box 2. Synthetic Drive}

Disease-transmitting insects impose a massive burden on human populations. There are an estimated 198 million cases of malaria each year, resulting in 580000 deaths, and 390 million people infected with dengue. Control of insect vectors using pesticides is expensive and can damage both ecosystems and people [84]. There is an urgent need for inexpensive, targeted pest control techniques. In recent years, researchers have turned to genetic engineering tools to control vectors of human disease with one of two goals: (i) to modify target populations to carry antipathogen genes that limit their capacity to spread disease, and (ii) to reduce or collapse target population sizes $[13,85]$. Various drive systems can be exploited to create synthetic drive systems (also known as gene drive) that can quickly spread through populations [85]. Transposable elements, homing endonucleases, Medea elements, Wolbachia, CRISPR-Cas9, as well as meiotic drivers each have potential use in synthetic drive methods to modify or collapse disease vector populations $[1,53,85-87]$.

Several groups have engineered synthetic drive systems in mosquitoes [88] and Drosophila [87,89,90]. Extreme sex ratio distortion offers one method of population extermination [13,53]. Galizi et al. [88] recently developed a homing endonuclease-based synthetic drive system capable of eliminating experimental populations of Anopheles gambiae mosquitoes (Figure I) within six generations by targeting $X$ chromosomes during meiosis. Alternative strategies for population modification or collapse involve synthetic toxin-antidote systems $[85,87,91]$. Many of these systems are modelled after Medea, a female gamete killing driver originally discovered in Tribolium castaneum that kills embryos that fail to inherit the element $[87,91]$.

Homing endonucleases have been used to create an artificial sperm killing meiotic drive system [85,88]. The new CRISPR-Cas9 genome editing technology targets specific sites in the genome and could prove to be a powerful source of synthetic drive systems, even in nonmodel pest species [86].

Synthetic drive systems have applications far beyond insect population control [92], including agriculture [93], controlling invasive species and pests, or even conservation [92]. We discuss the significant challenges and risks involved in the release of any such drive system in Box 3.

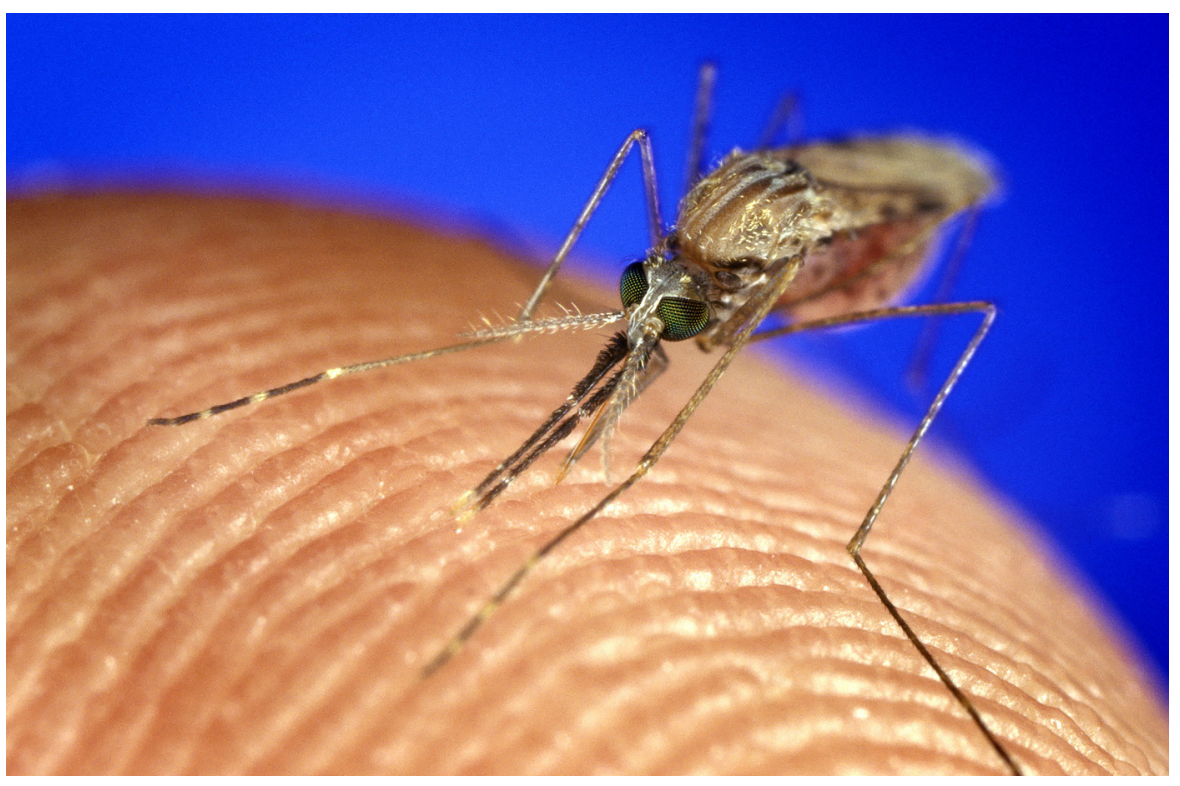

Trends in Ecology \& Evolution

Figure I. Anopheles gambiae Female. This is the primary species responsible for the transmission of Plasmodium falciparum - the parasite that causes malaria - to humans.

might also shape fundamental aspects of eukaryotic biology, including meiosis, gametogenesis, and genome structure [9-11]. Finally, understanding how ecology influences the population dynamics of meiotic drivers is important for predicting the success of synthetic drive elements, which are currently being engineered and applied to the management of vector populations of important human diseases ([12], Boxes 2 and 3). In this review, we consider the impacts drivers melanogaster that kills sperm that do not carry a copy of it.

\section{Segregation distortion: biased}

transmission to the next generation by the selfish action of a genetic element.

Sperm killer: a male meiotic driver that impairs development of sperm that do not carry it.

Spore killer: a meiotic driver in fungi that kills spores that do not carry a copy of it.

Synthetic drive systems: drivers that have been artificially engineered in the laboratory.

Target sequence: specific DNA sequence that is acted upon by another factor such as a driver or nuclease.

$\boldsymbol{t}$ haplotype distorter: an autosomal driver acting in the house mouse male that harms sperm that do not carry a copy of it.

Telomere: a region of repetitive DNA that caps the ends of chromosomes. $\mathrm{X}$ - (or Y)-linked driver: meiotic drive system located on a sex chromosome. 


\title{
Trends in Ecology \& Evolution
}

\begin{abstract}
Box 3. Synthetic Drive: Lessons from Natural Drive Systems
Genetic engineering of synthetic drive systems (Box 2) for release in natural populations has provoked controversy. If a synthetic driver spreads successfully, will it spread to nontarget populations or species? Will the drive mechanism interfere with key molecular pathways, resulting in unexpected phenotypic changes? Progress toward a synthetic drive system in a target disease vector has been slow owing to challenges in genetic engineering in nonmodel organisms. However, genome editing using the CRISPR-Cas9 system has the potential to rapidly accelerate the field. Several groups have suggested policy or protocols for releasing drive systems, but with these recent advances, additional discussion and regulation is urgently needed $[12,92,94,95]$. Here we outline several key challenges and concerns.
\end{abstract}

\section{(i) Adverse Effects of Synthetic Drive}

Before a synthetic drive system can be used in a natural population, extensive testing for unintended consequences and side effects (e.g., it does not transmit other pathogens, lead to higher bite rates from insect disease vectors, or have unanticipated effects on local ecology) is needed. Adverse phenotypic effects might be ameliorated by introducing another driver to reverse the effects of the initial driver [92,95].

\section{(ii) Risk of Cross-contamination}

This risk is presumably low for homing endonuclease genes [85] or CRISPR-Cas9-based drive systems or other sitespecific synthetic drivers, and could be reduced further by targeting specific sites limited to the intended species [92].

\section{(iii) Suppressors}

Any drive system must spread rapidly enough to be relevant to human disease, and before the system has time to evolve suppressors [85]. Multiple drivers with multiple targets is one possible solution to combat suppression [85].

\section{(iv) Environmental Heterogeneity}

Many natural drive systems show patchy or clinal distributions, indicating that costs of drivers vary between locations. Even strong synthetic drivers might be unable to penetrate all areas a target inhabits, potentially leaving reservoirs where suppression can evolve.

The parallels between synthetic and natural drivers make it likely that synthetic drive can be usefully informed by understanding the function and regulation of natural drive systems. In particular, suppressors are common in natural drive systems and can evolve rapidly [62]. Modified natural drive systems in both Drosophila [53] and mosquito species [96] faced difficulties from the rapid response of segregating suppressors in the population.

can have on the genomes, individuals, and populations that harbour them, and then discuss the factors that influence the dynamics of drivers in natural populations.

\section{Consequences of Drive}

Genomic Conflict

Meiotic drivers can pose a significant cost to the rest of the genome, which is then under selection for unlinked alleles that suppress drive and restore equal segregation. Consider a driving allele that resides on an $X$ chromosome in a species with heterogametic $(X Y)$ males. The driving $X$ causes $Y$-bearing sperm to die, such that the driving $X$ is transmitted to all offspring, who become daughters. The spread of the driving $X$ makes the population sex ratio increasingly female-biased, until lack of males causes population collapse and extinction [13]. It is easy to imagine that any $Y$ chromosome that resists drive will be favoured by selection [14], even if the driver is rare. Once the population sex ratio has become female-biased, classical Fisherian sex ratio selection will favour any autosomal mutation that suppresses drive [13,15]. Interestingly, a recent comparative study on tetrapods suggests that sex chromosome drive could account for the evolutionary pattern of species with male heterogamety exhibiting more female-biased adult sex ratios than species with female heterogamety [16].

Many drive systems consist of multiple drivers and suppressors, with several loci being involved with drive expression [17]. These systems suggest that the conflict does not end once a drive suppressor has evolved. Instead, enhancers linked to the original drive locus could evolve to restore drive, resuming the conflict. In this way, a drive system can cycle through periods of apparent drive and lack of drive resembling a coevolutionary arms race [18], resulting in a 


\section{Trends in Ecology \& Evolution}

complex genetic drive system. Recurrent coevolution between drivers and suppressors can contribute to the rapid evolution of genes, satellite DNA, and pathways whose functions might otherwise be expected to be conserved.

Rapid Divergence in Sequences, Genome Organisation, and Populations

Drive can contribute to DNA sequence evolution via selfish, driving nucleotide substitutions. For example, the meiotic drive gene Overdrive (GenBank: GA19777) of the fruit fly, Drosophila pseudoobscura bogotana, differs from the nondriving wild-type allele of its close relative, Drosophila pseudoobscura pseudoobscura, by seven nucleotide changes [19]. More often, drive seems to involve copy number variants: the Segregation Distorter system of Drosophila melanogaster involves a partial duplication of a protein-coding gene [20]; the $\boldsymbol{t}$ haplotype distorter system of the house mouse (Mus musculus) involves four tandemly duplicated genes [21]; copy number gain of the R2d distorter locus in house mice is associated with drive [22]; and the tandemly repeated, rapidly evolving, testis-expressed ampliconic genes of mammalian sex chromosomes are thought to result from recurrent arms races over gene dosage [23]. Such arms races do not necessarily occur between a driver and suppressors: different allelic variants of a meiotic driver can also compete against one another [24,25]. The rapid evolution of centromeres and centromeric proteins is particularly striking because these essential proteins are otherwise expected to be highly conserved [26]. Early speculation that female meiotic drive might be responsible for this rapid centromeric change is now supported by evidence in Mimulus monkeyflowers [27]. Finally, testis-expressed de novo genes often arise and spread to fixation but then, once fixed, degenerate into nonfunctional pseudogenes - a pattern suggestive of drive [28]. The recent identification of a young, rapidly evolving heterochromatin protein gene involved in a case of $\mathbf{X}$ chromosome drive in Drosophila simulans strongly supports this idea [29].

Drive can also have large-scale impacts on genome organisation and chromosome structure. Sperm killing meiotic drive elements often begin with just two loci - a driver and a target sequence, with the driver tightly linked to a resistant target to prevent the production of a suicide chromosome - subsequently driver and target may become elaborated via the recruitment of genetically linked enhancers. Such linked, coadapted gene complexes are expected to evolve in regions of low recombination and can become further protected from recombination by chromosomal inversions [30]. Reduced recombination associated with male drive has been found in Segregation Distorter [31], the $t$ haplotype [32], Spore killer [33], and Drosophila recens Sex-Ratio [34]. Female drive can involve dramatic changes in the quantity and sequence content of centromeric satellite DNA and proteins, as centromeres evolve to compete for access to primary oocytes and avoid relegation to the polar bodies, losing their chance for transmission (Box 1, $[11,35,36])$. Female drive can also favour the evolution of chromosome fusions or fissions, in which two fused centromeres experience a transmission rate different from that of nonfused ones, thus fuelling karyotype evolution [4]. As drive is usually exclusive to one sex, it accentuates intralocus sexual conflict [37]. Hence, a drive locus is expected to acquire genetically linked sexually antagonistic loci [38], potentially explaining the origin of sex chromosomes [39].

The combined effects of drive on DNA, genome, and karyotype evolution can lead to rapid divergence between populations and ultimately to speciation. For example, the fixation of alternative chromosome fusions in different populations can result in incompatible karyotypes that cause meiotic segregation problems in heterozygous individuals $[36,40]$. Recurrent drive and suppression can lead to cryptic drive systems, where fair meiosis has been restored within a species, but in a hybrid individual the dormant or suppressed drive elements can then spring into action $[5,41,42]$. Owing to reduced recombination and lack of homology, well-differentiated sex chromosomes are more susceptible to the invasion of drive elements. The recurrent fixation of cryptic drive systems on sex chromosomes might explain the prominent role of the X chromosome in the evolution of hybrid sterility in a wide range of species [42-44]. Cryptic drive systems 


\section{Trends in Ecology \& Evolution}

appear to contribute to reproductive isolation between populations and species of Drosophila [19,45], stalk-eyed flies [46], and yeasts [47].

\section{Growth and Persistence of Populations}

Drive can also have ecological consequences. Female-biased populations are expected to have higher per capita growth rates $[13,48]$. Although individuals carrying $\mathbf{X}$-linked drivers might leave fewer descendants than other members of their subpopulation that lack drivers, subpopulations containing an intermediate frequency of drivers might have faster population growth relative to driver-free subpopulations [48] and competing species [49]. Finally, a significant consequence of distorted sex ratios is the potential for population extinction attributable to the lack of one sex $[13,50,51]$, although definite evidence for such extinctions is almost entirely limited to lab populations [52-54].

\section{Dynamics of Drive}

Stability of Driver Frequencies in Natural Populations

All else being equal, drivers are predicted to increase in frequency as a result of biased transmission, and go to fixation. However, the spread of a driver can be limited by genetic suppressors, as well as fitness costs to carriers such as decreased fertility or viability [50]. Most of the known drive elements impose fitness costs on their carriers $[6,31,55]$, either as a result of direct pleiotropic effects of the driver on survival or reproductive success, production of a biased sex ratio (in the case of sex-linked drivers), or via deleterious mutations linked to the driver. The latter are expected to build up in drive systems located in genomic regions with reduced recombination (e.g., inversions). Genetic studies suggest that some well-studied drive systems apparently have persisted for considerable time (estimated ages: $t$ haplotype in mice ca. 2 Mya [56], Drosophila pseudoobscura Sex-Ratio ca. 1 Mya [57]). This long-term stability is surprising: a drive polymorphism is characterised by powerful selection on drivers and suppressors, and simple models suggest even a small change in drive or suppression strength can potentially lead rapidly to extinction or fixation. However, well-studied drivers in stable polymorphisms may represent a biased sample, if most drivers rapidly reach fixation or extinction, thereby becoming almost impossible to detect.

Fitness costs to individuals homozygous for the drive allele might help explain the persistence of some polymorphisms $[51,58,59]$. As autosomal drivers only benefit from transmission bias when in heterozygotes, they are most likely to be able to drive when rare. At higher frequencies, driver homozygotes become common, unmasking any recessive deleterious mutations linked to the drive allele. Processes that increase homozygote frequency, such as inbreeding, are predicted to reduce autosomal driver frequency [58]. The general prediction of an intermediate equilibrium for drivers with homozygous costs is borne out in some cases; for example, in yellow monkeyflowers, male and female fitness costs measured in the field together predict the observed frequency of a centromere-associated driver [59]. However, driver frequency in natural populations is often substantially lower than predicted by simple models based on homozygote fitness effects $[17,60]$.

Field studies of driver dynamics are rare, as few wild populations harbouring meiotic drivers have been repeatedly sampled $[24,54,61,62]$. Long-term studies of driver frequencies within populations are even rarer [60]. Several species show apparently stable clines in driver frequency [54,62], for example, the frequencies in Drosophila pseudoobscura populations across North America have remained unchanged for 70 years. By contrast, a strong decline of the house mouse $t$ haplotype frequency within one population was seen over 6 years [60]. There are also examples of rapidly spreading drivers. $\ln D$. simulans, a young $X$ driver originating in Africa has spread in the Middle East within the past 2 decades [62] while simultaneously decreasing in East Africa because of genetic suppression. The reasons for the stability of some drive systems, and 


\section{Trends in Ecology \& Evolution}

the rapid spread and decline of others, are poorly understood and a major focus of drive research.

\section{Sexual Selection against Driver-Carrying Individuals}

Male and female mating behaviour are predicted to influence driver dynamics. The costs associated with drive create a benefit to avoiding mating with individuals carrying a driver, and thus preferences against driver carriers are expected to evolve [63]. In stalk-eyed flies (Teleopsis dalmanni), females prefer to mate with males with larger eyespans, and driver-carrying males tend to have smaller eyespans $[64,65]$. In some house mouse populations, females carrying the $t$ haplotype discriminate against driver males in choice tests, although wild-type females show no preference $[66,67]$. However, as recombination is expected to break linkage between drive elements and traits that allow mate choice [63], with undetectable drivers predicted to rapidly outcompete detectable forms, premating discrimination against driver males might be uncommon [6]. Alternatively, as many sperm killers significantly reduce sperm numbers, females could potentially avoid drivers by preferentially discarding sperm from males transferring small ejaculates, as hinted by a study in $D$. simulans [68]. The production of driver-carrying progeny can also be avoided through sperm competition when females mate with multiple males, assuming drivercarrying males are poor sperm competitors [69]. Both theoretical models $[51,60,70]$ and empirical studies $[54,55,61,71-73]$ support the idea that gamete competition can reduce driver frequencies and limit the spread of male drivers under some conditions (see [51]). Indeed, the presence of drive elements can select for and lead to an increase in female mating frequency. If female mating rates are density-dependent [73], this could make drivers rare in denser populations.

Spatial Heterogeneity

Driver distribution varies across space and between habitats, and this aspect of natural drive systems might be important for the successful application of artificial drivers (Boxes 2 and 3). Drivers in mice and monkeyflowers vary in abundance between populations $[59,74]$. Segregation Distorter is typically found at very low frequencies in D. melanogaster [31], while two other Drosophila species show latitudinal clines in driver frequency across North America [54,61]. Driver frequency correlates negatively with the frequency of polyandry in these populations, supporting the hypothesis that polyandry impacts the success of drivers in nature. However, in Drosophila neotestacea, the environmental factor that best predicts the frequency of drivers is winter temperature [75], implying that drivers might be limited by elevated susceptibility to cold in driver carriers. Frequency of drivers in D. pseudoobscura can cycle yearly [76], suggesting more complex ecological interactions control driver abundance. Sperm killers can interact with other environmental factors that affect male fertility, such as high temperature [77]. It seems that variation in driver fitness between populations can result from interactions between environmental factors and the characteristics of populations harbouring drivers, potentially including differences between populations in deleterious genes linked to drive elements.

\section{Fixation and Extinction of Drivers}

Stable drive systems might be the exception, not the rule, with most drivers rapidly reaching fixation or extinction and becoming undetectable [50]. Population extinction is frequently predicted by simple models of sex chromosome drive [13,50,51]. It is difficult to measure the frequency of drive-mediated extinction because extinct populations leave no trace: while sampling wild D. neotestacea, Pinzone and Dyer [54] collected 175 flies from an isolated population, $91 \%$ of which were female; the following year only three flies were found at the same site, all driver-carrying females, and only one was inseminated. Laboratory experiments suggest that local extinctions are likely $[52,53]$. Local extinctions might allow drive to persist in a spatial mosaic where drive-related local extinctions are followed by rapid recolonisation from nearby sites [78]. Finding definitive evidence for such processes is very difficult, and the frequency at which such extinctions occur cannot typically be gauged. 


\section{Trends in Ecology \& Evolution}

\section{Box 4. Discovering Drive \\ Initial Detection}

Meiotic drive, both apparent and cryptic, affects patterns of phenotypic, genetic, and genomic variation. Although these patterns are generally not exclusive to drive, and thus are not definitive signatures, they provide valuable clues that drive might be present in a population or species. Polymorphic spore killer and sex chromosome sperm killer systems might even be detectable in natural populations, as they visibly affect spores within an ascus and sex ratios in progeny, respectively. Similarly, high genetic variance in fertility that is incompatible with mutation-selection balance models might suggest the presence of either autosomal sperm killers or costs associated with other balanced drive polymorphisms [97]. All forms of drive discussed here could be revealed as genetically localised transmission ratio distortion (TRD) in mapping populations or pedigrees, and, with sufficient sample sizes, gametic distortion might be statistically distinguishable from postzygotic (nondrive) distortion mechanisms [98]. Indeed, cryptic drive systems, in which a driver and suppressor have both gone to local fixation, are primarily detectable as aberrant phenomena (sterility, sex ratio, TRD, chromosomal abnormalities) in experimental hybrids between distinct populations or species. As genomic scans of variation become increasingly common, there will also undoubtedly be cases where selective sweeps or balanced inversion polymorphisms reflect natural selection via meiotic drive rather than via individual fitness [99].

\section{Validation}

Of course, none of these possible indications of meiotic drive are exclusively (or even most plausibly) explained by drive rather than other processes. Thus, the characterisation of new drive systems ideally includes both exclusion of alternative processes that can generate TRD, infertility, or other suggestive phenomena, and positive validation of a given drive mechanism. Validation can be fairly difficult for some systems and forms of drive, but is relatively accessible in others. New genomic technologies are likely to accelerate both validation and detection of drive. For example, deep sequencing of pooled sperm of $\mathrm{F} 1$ hybrids can directly determine gamete frequency prior to the confounding effects of fertilisation, and thus holds great promise as a tool for the detection and validation of autosomal sperm killer systems [100]. Broad application of such approaches will be the key to addressing general questions about the relative frequency of different types of drive in nature.

Autosomal male meiotic drivers, as well as chromosomal variants driving through female meiosis, might often fix without causing extinction. Thus, models predict a large number of cryptic drive systems that could potentially be revealed by crosses between populations (Box 4). However, population studies of autosomal drivers are so rare that the evidence is extremely limited. Moreover, the best-studied autosomal sperm killing meiotic driver [31] and female meiotic driver [59] are polymorphic within species, not fixed. Consequently, we do not know how common autosomal and female drive systems are, nor how often they reach fixation.

\section{Poorly Understood Dynamics in Many Systems}

The ecological dynamics of spore killers in fungi are little known. Although the system is increasingly understood at the genetic level $[32,77]$, the rarity of local resource competition makes the advantage they gain from drive obscure [78]. Ecological understanding of the dynamics of female drivers is also poor, with the exception of Mimulus monkeyflowers [59]. Finally, some documented sperm killer systems are more complex than any existing theoretical models. For example, Drosophila paramelanica has two driving $X$ chromosomes, a $Y$ that is susceptible to both, another $Y$ that is resistant to one of the drivers, and latitudinal differences between populations in the co-occurrence of drivers and $Y$ chromosomes [79]. Currently, little is known about how multiple drivers and resistance chromosomes coexist. Understanding factors that influence natural drive system dynamics is likely to be important to ensure the successful application of synthetic drive systems (Boxes 2 and 3).

\section{Summary and Concluding Remarks}

The potential for meiotic drive is probably high in all sexual organisms with a diploid phase, because the conflict over the transmission of homologous chromosomes in haploid gametes is nearly universal. Our understanding of the ecological and evolutionary dynamics of drive is surprisingly poor, even in well-studied systems. Nevertheless, some consistent themes stand out. Genetic suppression can evolve to neutralise drivers to the extent that the driver becomes undetectable, and this suppression can evolve and spread extremely rapidly [62]. Yet

\section{Outstanding Questions}

Despite involving key processes of life, our understanding of meiotic drive remains rudimentary. Here, we outline some key unresolved questions.

\section{How common is drive?}

Drive is the result of a fundamental conflict and potentially occurs in any diploid organism. Yet known drivers come from a limited range of species. Is it simply that drivers are rare? If so, why? Or do drivers usually persist for a very short time before reaching fixation or going extinct? Alternatively, are some taxa particularly susceptible to drive? Indeed, we have little understanding of how often novel mutations create drive. Why are so many of the detected drivers so strong, when theory suggests weak drive should be common? Is it simply that weak drive is difficult to detect?

\section{Drivers across space and time}

Despite decades of research, we lack data on how driver varies across time and space. Consequently, we do not know if drive is stable or cycles. We also do not know if drivers require a metapopulation for survival, nor what limits the spread of drivers between populations. Moreover, do drivers spread between hybridising species?

\section{Molecular mechanisms of drive}

We understand the genetic basis of very few drive systems. Are there general themes in the mechanisms? Do all gametic drive systems target similar pathways, or is each unique? Is the preponderance of drive systems in the Diptera (flies) attributable to some shared weakness in spermatogenesis that drive can exploit? Why is genetic suppression apparently absent in some ancient drive systems? Do these drive systems target something fundamental that cannot be defended, or are these drivers simply evolving faster than their targets?

\section{Contrasting synthetic and natural} drive

How similar are the mechanisms of natural drive to synthetic drive systems? As the survivors of generations of counter selection, are natural drivers more robust than synthetic ones? Or are they limited by mutations where the designers of synthetic drivers are not? 


\section{Trends in Ecology \& Evolution}

suppression is not universal, and some ancient systems seem to have never evolved resistance or suppression. All well-studied extant drivers have costs, either intrinsic to the mechanism they use to gain their transmission advantage, or resulting from the reduced recombination that commonly associates with drive. Repeated discoveries of such associations suggest that extant drive systems are often complex, using multiple genes, perhaps indicating that successful drivers need modifiers that help them avoid suppression. Active drive systems vary in frequency between populations, and sometimes over seasons and years, suggesting that the fitness of drivers depends on their local environmental conditions, in ways that are currently not well understood.

Novel synthetic drive techniques (Box 2) have the potential to fundamentally alter natural populations in ways analogous to meiotic drive. These synthetic drive systems have enormous potential for biocontrol, but if they are used without understanding how drive behaves in natural systems, there are serious risks of synthetic drive both failing to achieve its aims and having unintended negative consequences. Work on natural drive systems shows that the consequences of drive are manifold, from speciation to genome organisation, gametogenesis, competition among species, mate choice, and mating systems. Once synthetic drivers are released into nature, the potential for long-term evolutionary changes in the target species and its community are profound.

New natural drive systems will be discovered in coming years (Box 4), for example, by the discovery of non-Mendelian patterns of inheritance in sequence data. Detecting new drivers should help answer many of the outstanding questions in the field (see Outstanding Questions), and without doubt will uncover new mechanisms of drive, as well as unexpected genomic consequences of drive.

\section{Acknowledgments}

This review was written at the Ecology of Meiotic Drive workshop, with funding from the Swiss National Science Foundation (IZ32ZO_160288), the Russian Science Foundation (15-29-02550), the Department of Evolutionary Biology and Environmental Studies (University of Zurich), and the Vereinigung akademischer Mittelbau der Universität Zürich.

\section{References}

1. Sandler, L. and Novitski, E. (1957) Meiotic drive as an evolutionary force. Am. Nat. 91, 105-110

2. Haldane, J.B.S. (1932) The Causes of Evolution, Longmans, Green and Co

3. Bull, J.J. (2015) Evolutionary decay and the prospects for longterm disease intervention using engineered insect vectors. Evol. Med. Public Health 2015, 152-166

4. Pardo-Manuel De Villena, F. and Sapienza, C. (2001) Nonrandom segregation during meiosis: the unfairness of females. Mamm. Genome 12, 331-339

5. Presgraves, D. (2009) Drive and sperm: the evolution and genetics of male meiotic drive. In Sperm Biology: An Evolutionary Perspective (Birkhead, T.R et al, eds), pp. 471-506, Elsevier

6. Price, T.A.R. and Wedell, N. (2008) Selfish genetic elements and sexual selection: their impact on male fertility. Genetica 134,99-111

7. Raju, N.B. (1994) Ascomycete spore killers: chromosomal elements that distort genetic ratios among the products of meiosis. Mycologia 86, 461-473

8. Crespi, B. and Nosil, P. (2013) Conflictual speciation: species formation via genomic conflict. Trends Ecol. Evol. 28, 48-57

9. Hurst, G.D.D. and Werren, J.H. (2001) The role of selfish genetic elements in eukaryotic evolution. Nat. Rev. Genet. 2, 597-606

10. Rice, W.R. (2013) Nothing in genetics makes sense except in light of genomic conflict. Annu. Rev. Ecol. Evol. Syst. 44, 217-237

11. Henikoff, S. et al. (2001) The centromere paradox: stable inheritance with rapidly evolving DNA. Science 293, 1098-1102

12. Oye, K.A. et al. (2014) Regulating gene drives. Science 345 , $626-628$
13. Hamilton, W. (1967) Extraordinary sex ratios. Science 156 477-488

14. Unckless, R.L. et al. (2015) Sex-Ratio meiotic drive and Y-linked resistance in Drosophila affinis. Genetics 199, 831-840

15. Fisher, R.A. (1930) The Genetical Theory of Natural Selection The Clarendon Press

16. Pipoly, I. et al. (2015) The genetic sex-determination system predicts adult sex ratios in tetrapods. Nature 527, 91-94

17. Jaenike, J. (2001) Sex chromosome meiotic drive. Annu. Rev. Ecol. Evol. Syst. 32, 25-49

18. Hall, D.W. (2004) Meiotic drive and sex chromosome cycling Evolution 58, 925-931

19. Phadnis, N. and Orr, H.A. (2009) A single gene causes both male sterility and segregation distortion in Drosophila hybrids. Science 323, 376-379

20. Merrill, C. et al. (1999) Truncated RanGAP encoded by the Segregation Distorter locus of Drosophila. Science 283, 1742-1745

21. Bauer, H. et al. (2005) The $t$-complex-encoded GTPase-activating protein Tagap1 acts as a transmission ratio distorter in mice. Nat. Genet. 37, 969-973

22. Didion, J.P. et al. (2015) A multi-megabase copy number gain causes maternal transmission ratio distortion on mouse chromosome 2. PLoS Genet. 11, e1004850

23. Nam, K. et al. (2015) Extreme selective sweeps independently targeted the X chromosomes of the great apes. Proc. Natl. Acad. Sci. U.S.A. 112, 6413-6418

24. Brand, C.L. et al. (2015) Origin, evolution, and population genetics of the selfish Segregation Distorter gene duplication in

\section{Evolutionary impacts of drive}

Theory suggests drive has major impacts on meiosis and gametogenesis, and may be a major reason for recombination itself. Has drive really had this much impact? Drive has also been proposed as a mechanism for promoting speciation by rapidly generating idiosyncratic differences between populations in reproductive genes, but the evidence is not yet conclusive. Finally, does drive really cause population or even species extinctions, and if so has this species-level selection impacted traits in extant organisms? 


\section{Trends in Ecology \& Evolution}

European and African populations of Drosophila melanogaster. Evolution 69, 1271-1283

25. van Boven, M. and Weissing, F.J. (2001) Competition at the mouse $t$ complex: rare alleles are inherently favored. Theor. Popul. Biol. 60, 343-358

26. Malik, H.S. and Henikoff, S. (2009) Major evolutionary transitions in centromere complexity. Cell 138, 1067-1082

27. Finseth, F.R. et al. (2015) Duplication and adaptive evolution of a key centromeric protein in Mimulus, a genus with female meiotic drive. Mol. Biol. Evol. 32, 2694-2706

28. Bai, Y. et al. (2007) Comparative genomics reveals a constant rate of origination and convergent acquisition of functional retrogenes in Drosophila. Genome Biol. 8, R11

29. Helleu, Q. et al. Rapid evolution of a Y-chromosome heterochromatin protein underlies sex chromosome meiotic drive. Proc. Natl. Acad. Sci. U.S.A. (in press)

30. Charlesworth, B. and Hartl, D.L. (1978) Population dynamics of the Segregation Distorter polymorphism of Drosophila melanogaster. Genetics 89, 171-192

31. Larracuente, A.M. and Presgraves, D.C. (2012) The selfish Segregation Distorter gene complex of Drosophila melanogaster. Genetics 192, 33-53

32. Justice, M.J. and Bode, V.C. (1988) Genetic analysis of mouse $t$ haplotypes using mutations induced by ethylnitrosourea mutagenesis: the order of $T$ and $q k$ is inverted in $t$ mutants. Genetics 120, 533-543

33. Harvey, A.M. et al. (2014) A critical component of meiotic drive in Neurospora is located near a chromosome rearrangement. Genetics 197, 1165-1174

34. Dyer, K.A. et al. (2007) Chromosome-wide linkage disequilibrium as a consequence of meiotic drive. Proc. Natl. Acad. Sci. U.S.A. 104, 1587-1592

35. Fishman, L. and Saunders, A. (2008) Centromere-associated female meiotic drive entails male fitness costs in monkeyflowers. Science 322, 1559-1562

36. Chmátal, L. et al. (2014) Centromere strength provides the cell biological basis for meiotic drive and karyotype evolution in mice. Curr. Biol. 24, 2295-2300

37. Manser, A. et al. (2012) The effect of polyandry on a distorter system with differential viabilities in the sexes. Commun. Integr. Biol. 5, 550-552

38. Patten, M.M. (2014) Meiotic drive influences the outcome of sexually antagonistic selection at a linked locus. J. Evol. Biol. 27, 2360-2370

39. Kozielska, M. et al. (2010) Segregation distortion and the evolution of sex-determining mechanisms. Heredity 104, 100-112

40. Baker, R.J. and Bickham, J.W. (1986) Speciation by monobrachial centric fusions. Proc. Natl. Acad. Sci. U.S.A. 83, 82458248

41. Frank, S.A. (1991) Divergence of meiotic drive-suppression systems as an explanation for sex-biased hybrid sterility and inviability. Evolution 45, 262-267

42. Hurst, L.D. and Pomiankowski, A. (1991) Causes of sex ratio bias may account for unisexual sterility in hybrids: a new explanation of Haldane's rule and related phenomena. Genetics 128, $841-$ 858

43. Presgraves, D.C. (2010) The molecular evolutionary basis of species formation. Nat. Rev. Genet. 11, 175-180

44. Meiklejohn, C.D. and Tao, Y. (2010) Genetic conflict and sex chromosome evolution. Trends Ecol. Evol. 25, 215-223

45. Zhang, L. et al. (2015) Sex ratio meiotic drive as a plausible evolutionary mechanism for hybrid male sterility. PLoS Genet. 11, e1005073

46. Wilkinson, G.S. et al. (2014) Haldane's Rule is linked to extraordinary sex ratios and sperm length in stalk-eyed flies. Genetics $198,1167-1181$

47. Zanders, S.E. et al. (2014) Genome rearrangements and pervasive meiotic drive cause hybrid infertility in fission yeast. Elife 3 , e02630

48. Policansky, D. (1974) 'Sex Ratio,' meiotic drive, and group selection in Drosophila pseudoobscura. Am. Nat. 108, 75-90
49. Unckless, R.L. and Clark, A.G. (2014) Sex-ratio meiotic drive and interspecific competition. J. Evol. Biol. 27, 1513-1521

50. Carvalho, A.B. and Vaz, S.C. (1999) Are Drosophila SR drive chromosomes always balanced? Heredity 83, 221-228

51. Holman, L. et al. (2015) Coevolutionary dynamics of polyandry and sex-linked meiotic drive. Evolution 69, 709-720

52. Price, T.A.R. et al. (2010) Polyandry prevents extinction. Curr Biol. 20, 1-5

53. Lyttle, T.W. (1977) Experimental population genetics of meiotic drive systems I. Pseudo-Y chromosomal drive as a means of eliminating cage populations of Drosophila melanogaster. Genetics 86, 413-445

54. Pinzone, C.A. and Dyer, K.A. (2013) Association of polyandry and sex-ratio drive prevalence in natural populations of Drosophila neotestacea. Proc. Biol. Sci. 280, 20131397

55. Sutter, A. and Lindholm, A.K. (2015) Detrimental effects of an autosomal selfish genetic element on sperm competitiveness in house mice. Proc. Biol. Sci. 282, 20150974

56. Silver, L.M. (1993) The peculiar journey of a selfish chromosome: mouse $t$ haplotypes and meiotic drive. Trends Genet. 9 , 250-254

57. Kovacevic, M. and Schaeffer, S.W. (2000) Molecular population genetics of X-linked genes in Drosophila pseudoobscura. Genetics 156, 155-172

58. Lewontin, R.C. and Dunn, L.C. (1960) The evolutionary dynamics of a polymorphism in the house mouse. Genetics 45, 705-722

59. Fishman, L. and Kelly, J.K. (2015) Centromere-associated meiotic drive and female fitness variation in Mimulus. Evolution 69, 1208-1218

60. Manser, A. et al. (2011) Polyandry and the decrease of a selfish genetic element in a wild house mouse population. Evolution 65 2435-2447

61. Price, T.A.R. et al. (2014) Does polyandry control population sex ratio via regulation of a selfish gene? Proc. Biol. Sci. 281 20133259

62. Bastide, H. et al. (2013) Local dynamics of a fast-evolving sexratio system in Drosophila simulans. Mol. Ecol. 22, 5352-5367

63. Lande, R. and Wilkinson, G.S. (1999) Models of sex-ratio meiotic drive and sexual selection in stalk-eyed flies. Genet. Res. 74 245-253

64. Johns, P.M. et al. (2005) Genetic linkage between a sexually selected trait and X chromosome meiotic drive. Proc. Biol. Sci. 272, 2097-2103

65. Cotton, A.J. et al. (2014) Male eyespan size is associated with meiotic drive in wild stalk-eyed flies (Teleopsis dalmanni). Heredity 112, 363-369

66. Lenington, S. et al. (1994) Female preference and variability among t-haplotypes in wild house mice. Am. Nat. 143, 766-784

67. Manser, A et al (2015) Female house mice avoid fertilization by t haplotype incompatible males in a mate choice experiment. $J$. Evol. Biol. 28, 54-64

68. Angelard, C. et al. (2008) Female-driven mechanisms, ejaculate size and quality contribute to the lower fertility of sex-ratio distorter males in Drosophila simulans. BMC Evol. Biol. 8, 326

69. Haig, D. and Bergstrom, C.T. (1995) Multiple mating, sperm competition and meiotic drive. J. Evol. Biol. 8, 265-282

70. Taylor, J.E. and Jaenike, J. (2002) Sperm competition and the dynamics of $X$ chromosome drive: stability and extinction. Genet ics 160, 1721-1731

71. Price, T.A.R. et al. (2008) Sex ratio distorter reduces sperm competitive ability in an insect. Evolution 62, 1644-1652

72. Wilkinson, G.S. and Fry, C.L. (2001) Meiotic drive alters sperm competitive ability in stalk-eyed flies. Proc. Biol. Sci. 268, 2559 2564

73. Atlan, A. et al. (2004) Sex-ratio distorter of Drosophila simulans reduces male productivity and sperm competition ability. J. Evol. Biol. 17, 744-751

74. Safronova, L. et al. (2010) The expression of transmission ratio distortion (TRD) and the frequency of carriers of $t$ haplotypes in natural populations of house mice Mus musculus. Russ. J. Genet. 46, 578-585 


\section{Trends in Ecology \& Evolution}

75. Dyer, K.A. (2012) Local selection underlies the geographic distribution of sex-ratio drive in Drosophila neotestacea. Evolution 66, 973-984

76. Bryant, S.H. et al. (1982) 'Sex-ratio' trait, sex composition, and relative abundance in Drosophila pseudoobscura. Evolution 36 , 27-34

77. Price, T.A.R. et al. (2012) No evidence that temperature-related fertility differences influence the distribution of a selfish genetic element. Funct. Ecol. 26, 657-665

78. van Boven, M. and Weissing, F.J. (1999) Segregation distortion in a deme-structured population: opposing demands of gene, individual, and group selection. J. Evol. Biol. 12, 80-93

79. Stalker, H.D. (1961) The genetic systems modifying meiotic drive in Drosophila paramelanica. Genetics 46, 177-202

80. Buckler, E.S. et al. (1999) Meiotic drive of chromosomal knobs reshaped the maize genome. Genetics 153, 415-426

81. Taylor, D.R. (1994) The genetic basis of sex ratio in Silene alba (= S. latifolia). Genetics 136, 641-651

82. Presgraves, D.C. et al. (1997) Sex chromosome meiotic drive in stalk-eyed flies. Genetics 147, 1169-1180

83. Grognet, P. et al. (2014) Genes that bias Mendelian segregation. PLoS Genet. 10, e1004387

84. Pimentel, D. and Burgess, M. (2014) Environmental and economic costs of the application of pesticides primarily in the United States. In Integrated Pest Management. Pesticide Problems (Pimentel, D. and Peshin, R., eds), pp. 47-71, Springer

85. Burt, A. (2014) Heritable strategies for controlling insect vectors of disease. Philos. Trans. R. Soc. B: Biol. Sci. 369, 20130432

86. Gantz, V.M. et al. (2015) Highly efficient Cas9-mediated gene drive for population modification of the malaria vector mosquito Anopheles stephensi. Proc. Natl. Acad. Sci. U.S.A. 112, E6736E6743

87. Chen, C-H. et al. (2007) A synthetic maternal-effect selfish genetic element drives population replacement in Drosophila. Science 316, 597-600
88. Galizi, R. et al. (2014) A synthetic sex ratio distortion system for the control of the human malaria mosquito. Nat. Commun. 5 , 4977

89. Chan, Y.S. et al. (2011) Insect population control by homing endonuclease-based gene drive: an evaluation in Drosophila melanogaster. Genetics 188, 33-44

90. Simoni, A. et al. (2014) Development of synthetic selfish elements based on modular nucleases in Drosophila melanogaster. Nucleic Acids Res. 42, 7461-7472

91. Hay, B.A. et al. (2010) Engineering the genomes of wild insect populations: challenges, and opportunities provided by synthetic Medea selfish genetic elements. J. Insect Physiol. 56, 14021413

92. Esvelt, K.M. et al. (2014) Concerning RNA-guided gene drives for the alteration of wild populations. Elife 3, e03401

93. Houben, A. et al. (2008) Engineered plant minichromosomes: bottom-up success? Plant Cell 20, 8-10

94. Akbari, O.S. et al. (2015) Safeguarding gene drive experiments in the laboratory. Science 349, 927-929

95. DiCarlo, J.E. et al. (2015) Safeguarding CRISPR-Cas9 gene drives in yeast. Nat. Biotechnol. 33, 1250-1255

96. Hickey, W.A. and Craig, G.B., Jr (1966) Distortion of sex ratio in populations of Aedes aegypti. Can. J. Genet. Cytol. 8, 260-278

97. Scoville, A. et al. (2009) Contribution of chromosomal polymorphisms to the G-matrix of Mimulus guttatus. New Phytol. 183, 803-815

98. Knief. U. et al. (2015) A prezygotic transmission distorter acting equally in female and male zebra finches Taeniopygia guttata. Mol. ECol. 24, 3846-3859

99. Garrigan, D. et al. (2014) Genome diversity and divergence in Drosophila mauritiana: multiple signatures of faster $\mathrm{X}$ evolution. Genome Biol. Evol. 6, 2444-2458

100. Corbett-Detig, R. et al. (2015) Direct gamete sequencing reveals no evidence for segregation distortion in house mouse hybrids. PLOS ONE 10, e0131933 